\title{
ANTHRACYCLINE CARDIOMYOPATHY IN ANESTHESIOLOGY
}

\section{Andrei Lezhnev ${ }^{凶}$, Viktor Paramonov, Oleg Solontsov, Pavel Shchennikov, Dmitry Davydov, Aleksandr Davydov, Ruslan Bikbaev}

Clinic of Dr. Paramonov, Saratov, Russia

klinika@dr-paramonov.ru

ABSTRACT - The review covers the formation of clinical forms and manifestations of the mutual influence of carcinogenesis, chemotherapy and concomitant cardiovascular diseases, mechanisms of heart failure development and ways of its medical correction.

KEY W ORDS - cardiotoxicity, carcinogenesis, anthracyclines, endothelial dysfunction, cytoprotection.

As a result of a targeted cancer therapy which has led to an increase in the number of patients who have survived, the frequency of use and intensity of chemotherapy, there are conditions for the formation of chemotherapy complications, the most important of which is the myocardial damage. [1,2]. Patients began to "live" until the development of such delayed complications as cardiomyopathy and congestive heart failure $(\mathrm{CHF})$.

An anesthesiologist at a general surgery clinic sees an increasing number of patients who have previously undergone chemotherapy treatment. In our opinion, the chemotherapeutic history does not receive proper attention. However, these data may be critical for the assessment of the functional operability and prevention of cardiovascular complications during the perioperative period in this category of patients..

\section{The objective of the review}

is to describe the mechanisms of the cardiotoxicity development in combination with the pathogenesis of the oncological process, chemotherapy and concomitant cardiovascular diseases as well as to find ways to reduce cardiac risks in general surgical practice.

The most commonly used drugs in chemotherapy are anthracycline antibiotics. Anthracycline-induced cardiomyopathy (AC-CMP) is a malignant form of heart disease with a mortality rate of up to $50 \%$ during the first 2 years after chemotherapy [3]. The therapeutic efficiency of anthracyclines is high and dose- dependent. The dose-dependency is traced not only in the therapeutic effect of the drug, but also in its toxic effects [4].

The predictors of the cardiotoxicity are the cumulative dose, age and concomitant cardiovascular pathology, as well as the duration of treatment [5]. The developing heart is particularly vulnerable; children who receive anthracyclines are at an extremely high risk of developing an anthracycline-induced cardiotoxicity. A study by Cardinale et al, involving 2,625 patients (the mean follow-up time of 5.2 years), found cardiotoxicity in $9 \%$ of cases after an anthracycline treatment, with $98 \%$ of cases occurring during the first year and being asymptomatic. A 15 -fold increase in the risk of developing heart failure was found in those who had undergone cancer treatment in childhood [6].

Cardiologists distinguish between the following forms of cardiotoxicity: symptomatic cardiotoxicity is found in 2-4\% and asymptomatic reduction of LVEF in $9-11 \%$ of patients. However, the increase of cardiac biomarkers in patients who have undergone chemotherapy was noted in $30-35 \%$ of patients. [5].

The spectrum of complications in the cardiovascular system as a result of an anticancer therapy is not limited to cardiac pathology. Arterial hypertension, thromboembolic complications, peripheral vascular diseases, strokes, pericarditis are additional probable components of risk conditions in this category of patients [7].

The pathophysiological picture in cancer patients is not a simple sum of the changes in the systems, but rather a complex superposition of tumor development, antitumor treatment and related conditions. The cancer process has a multidimensional effect on the body, including mechanical compression of surrounding structures by the tumor, invasion of the pathological agent into the surrounding tissues and a polysystemic effect on the body. On the other hand, chemotherapy is not only aggressive towards tumor cells, but also towards all cellular systems. When these processes are imposed on the concomitant states and their medication, not only the differences in pathogenetic mechanisms, but also their coincidences are determined. The scope of the review includes the effects on the most vulnerable system, the cardiovascular system.

The main antitumor mechanism of the antracycline action is implemented through the blockade of 
the topoisomerase (Top) system, the enzyme system responsible for the cell division. Top $2 \alpha$ is the main molecular target of the antitumor activity of anthracycline. Top $2 \beta$ is subjected to exposure while it is in cardiomyocytes $[8,9]$.

The realisation of the direct cardiotoxic influence of anthracyclines through topoisomerase is combined with the indirect influence. Cardiomyocyte apoptosis is mediated by the release of reactive oxygen species (ROS) and oxidative stress [2], mitochondrial and endothelial dysfunction. The calcium overload is also involved in the formation of the ROS pool [5] and in the blockade of the mitochondrial pore [10]. The mitochondrial pore is a channel that plays a significant role in the calcium metabolism between the mitochondria and the medium and can initiate the process of cellular degradation. Nitrogen oxide may act as a modulator of the mitochondrial pore opening (here, the intersection with the endothelial dysfunction process). When the apoptosis activators leave the mitochondria, the cell destruction is triggered.

Doxorubicin-iron complexes are the second pathway of the formation of toxic radicals leading to an increase in nitrosative stress and mitochondrial dysfunction [8].

A consensus of oncologists discusses the mechanisms of cardiomyopathy development after chemotherapy [6]. The most important pathophysiological mechanisms are: suppression of vascular endothelium growth factor ejection, nitrogen oxide synthesis, vascular rarefaction (reduction), oxidative stress, renal thrombotic microangiopathy, hypercoagulation, direct endothelial damage. Coronary spasms and arterial thrombosis form ischemic syndromes: arrhythmia, ACS, sudden death. Endothelium (E) is one of the cellular systems most sensitive to the influences. During the AC-CMP formation, a depression of the nitrogen oxide formation leads to a decrease in the vascular dilation reserve, $\beta$-adrenergic inotropic and chronotropic cardiomyocyte responses [11].

Thus, direct and delayed destructive cardiotoxic effects of chemotherapy form the risk of cardiovascular complications. Direct effects on cardiomyocytes are supplemented by indirect pathogenic processes caused not only by the chemotherapy medication, but also by the oncological process itself.

\section{MECHANISMS OF CARDIOTOXICITY OF CARCINOGENESIS}

The cancerous process, even in the absence of mechanical compression of tissue due to the extra volume, causes severe body disorders in the form of tumour intoxication. Toxic manifestations can be characterized by various syndrome complexes: anorexia-kahexia, asthenisation, tumour decomposition syndromes, hemolytic-uremic syndrome, paraneoplastic syndrome, etc. [12]. Common for the cancer process of any localisation is a combination of anemia, energy deficiency, hemostasis disorders...

Anemia is an independent negative prognostic factor of the tumor growth. A $1 \%$ decrease in oxygen content stimulates the development of transcription factors for responsible genes. Tumor cell adaptation to anemia stimulates angiogenesis, blocks apoptosis and promotes tumor growth, invasion and metastasis. A low oxygen content in the cell is a factor of chemoand radio-resistance of the tumor [12].

Anemia is responsible not only for the degree of malignancy of the tumor cell, but also for its energy deficiency. Nutritional disturbances, as an element of the cancerous intoxication, occur after restructuring the metabolism of tumor cells in combination with anemia, and cause deficiency of many substrates (amino acids, vitamins, trace elements) [13].

In terms of the postoperative prognosis, special attention is paid to the influence of tumor cells on the hemostasis system. The pathogenesis of thrombotic complications consists of a combination of disorders caused by the tumor and the treatment. Blood coagulation activation mechanisms include: isolation of a highly active tissue factor and procoagulant by tumor cells, increase of procoagulant activity of monocytes, platelets and endothelial cells in response to the tumor growth [14].

In practice, it is not the thromboembolic complications (TECs) that are more frequently dealt with, but rather their risks. Risk factors for TECs in both surgical patients and patients receiving antitumor therapy do not differ much from the common ones: clinical (age over 40, infection, TEC in the patient's history, varicosity, heart and vascular diseases, kidney diseasse, obesity, etc.), hypercoagulation and thrombocytosis, leukocytosis, anemia, erythropoietin use [15].

The tumor process itself, being an arrhythmogenic process, increases the likelihood of the TEC onset [16].

The influence of factors related to the endothelium on the malignancy of the tumor has been proven. The lowest levels of the angiogenic inhibitor, endostatin, have been reported in patients with ovarian cancer. The vascular endothelial growth factor (VEGF), an angiogenesis activator, was three times higher in the malignant process than in the benign process and six times higher in comparison with the control group. In other words, it is necessary to consider the endostatin and the VEGF levels as malignancy factors in ovarian cancer [17].

On the one hand, there is the activation of neoangiogenesis, hemostatic balance stress, anemia and 
energy deficiency. On the other hand, there is a critical increase in the pool of aggressive compounds and radicals, mitochondrial insufficiency and disturbance of nitrogen oxide homeostasis. These are the main components of the pathogenesis of the tumor process, which is subject to chemotherapy. An endothelial dysfunction is common for the above mentioned processes of complex mutual influences of tumor growth and chemotherapy. A tumor process, combined with a therapeutic effect, leads to tension, hyperfunction and exhaustion of the endothelium system.

Cardiovascular pathology associated with an oncological process, being both the cause and result of the endothelial dysfunction, [18] complements the pathogenetic picture.

Thus, the conditions of the endothelial dysfunction formation in the considered category of patients is an integral factor of mutual influence of the processes of tumor growth, chemotherapy and concomitant pathology.

\section{CARDIAL RISKS,}

THEIR PREVENTION AND WAYS

OF REDUCTION IN AC-CMP

When examined by an anesthesiologist in general surgery, patients in this group are often either denied surgical care as "hopeless" or do not receive a proper assessment of the cardiovascular system with a risk of postoperative fatal events. The assessment of cardiovascular risk factors and the prevention and reduction of these risks are of particular importance.

Cardiac risk factors (over 65 years of age, cardiovascular pathology) in the development of AC-CMP are supplemented by a previous radiation exposure of the mediastinal area in the course of chemotherapy, cumulative dose of chemotherapy medication, as well as the total dose administered per day or per course of chemotherapy, the rate of drug administration, the female sex and electrolyte imbalance [19]. In the long run, the cardiovascular risk factors become cardiac in nature. The main information which draws the attention of an anesthesiologist when working with this group of patients is cardiac anamnesis. Thus, the main argument in the correction of risks is the adherence and sequence of the cardiac therapy.

Currently, there is no consensus in regard to the control of antacycline-induced cardiotoxicity [9]. The lack of highly effective specific treatment of the anthracycline-induced heart failure sends us back to the standard treatment of the congestive heart failure with ACE inhibitors, beta-blockers, and looped diuretics [20].

The traditional cardiac strategy is complemented by the cytoprotectors reamberin [21], carvedilol [22], and ivabradine [23].
In terms of the correction of the endothelial dysfunction, statins are of particular interest in the treatment of AC-CMP. In cases with melanoma, colorectal and lung carcinoma, lovastatin demonstrated not only a decrease in the risk of doxorubicin-induced cardiotoxicity, but also an increase in the antitumor effect of the chemotherapy medication [24]. Preclinical experimental models of breast cancer showed antineoplastic activity in statins [25]. Antitumor effects of statins are associated with their ability to slow down tumor cell growth, induce apoptosis, reduce its metastatic potential, and inhibit angiogenesis [26]. The known hypolipidemic and pleiotropic effects of statins determine the possible potential in the prevention and treatment of myocardial injuries during the treatment with antitumor drugs, without affecting the dynamics of the carcinogenesis [27].

The use of Dexrazoxane (Cardioxane) is questionable, given the common target with antitumor drugs. However, to some extent, its effect can be regarded as a specific cardioprotective one. In the Cochrane meta-analysis of adult cancer patients receiving anthracyclines, Dexrazoxane significantly reduced the risk of the heart failure development without significant differences from the control group in terms of efficacy and survival [6].

Therefore, the preparation for chemotherapy should include examination of the cardiovascular system before and during the treatment, and chemotherapy should be accompanied by a continuous cardiac treatment.

\section{CONCLUSIONS}

The anesthesiologist may see patients months and years after their chemotherapy. During this time, heart failure of varying degrees of severity is formed. A large risk group of patients with maximum cardiotoxicity and severe general surgery prognosis are those who received a prolonged chemotherapy treatment in childhood. The mechanisms of the myocardiocytes damage are difficult to correct, which urgently requires the inclusion of cardioprotective drugs in the chemotherapy treatment and in the follow-up period. The targets and methods of chemotherapy contradict the development of cardiovascular complications: the majority of authors confirm the dependence of cardiovascular complications formation frequency on the total dose of the chemotherapy drugs. The effectiveness of therapy also depends on the cumulation. Contradictions can be resolved by using endothelial protection drugs and statins.

When prescribing treatment for cancer patients, the interaction of a cardiologist and an oncologist is crucial. It is important to study the subject of the car- 
diovascular therapy as a component of the antitumor treatment.

\section{REFERENCES}

1. Minimum clinical recommendations of the European Society of Medical Oncology (ESMO). Editors of the Russian translation: Prof. S.A. Tyulyandin, Candidate of Medical Sciences D.A. Nosov; Prof. N.I. Translatorova. M.: The Blokhin Russian Academy of Medical Sciences Publishing Group, 2010, 436 pages;

2. Anthracycline Chemotherapy and Cardiotoxicity. John V McGowan, Robin Chung, Angshuman Maulik, Izabela Piotrowska, J. MalcolmWalker, Derek M. Yellon// Cardiovasc. Drugs Ther (2017) 31:63-75. DOI 10.1007/s10557-016-6711-0;

3. Rickard J., Kumbhani D.J., Baranowski B., MarTin D.O., Tang W.H., Wilkoff B.L.: Usefulness of cardiac resynchronization therapy in patients with Adriamycin-induced cardiomyopathy, Am J Cardiol; 2010, feb. 15, 105 (4): 522-526;

4. L.V. Bolotina, A.G. Ovchinnikova: Problems of cardiovascular complications induced by chemotherapy and targeted drugs / / Studies and practice in medicine. 2015, vol. 2, No. 4, pp. 106-114;

5. Cardiovascular prophylaxis 2017. Russian national recommendations // Russian Journal of Cardiology. 2018; 23(6): 7-122. http://doi.org/10.15829/15604071-2018-6-7-122

6. 2016 ESC Position Paper on cancer treatments and cardiovascular toxicity developed under the auspices of the ESC Committee for Practice Guidelines // Russian Journal of Cardiology, 2017, №3 (143): pp. 105-139. http://doi.org/10.15829/1560-4071-2017-3-105-139

7. R.A. YaNdieva, E.K. SARIBEKYan, M.N. MamMADOV: Cardiotoxicity in the treatment of cancer // Journal of Heart and Vascular Diseases. Vol. 6, No. 17, 2018, pp. 3-11;

8. G. E. Gendlin, E. I. Emelina, I. G. Nikitin, Y. A. VASYUK: Modern view on cardiotoxicity of chemotherapy of oncological diseases, including anthracycline antibiotics. // Russian Journal of Cardiology. 2017, 3 (143): pp. 145-154;

9. HeNriksen P.A. Anthracycline cardiotoxicity: an update on mechanisms, monitoring and prevention. Heart, 2018; 104: pp. 971-977;

10. E. V. Pozhilova, O. S. Levchenkova, V. E. NoVIKOV: The regulatory role of the mitochondrial pore and the possibilities of its pharmacological modulation // Reviews on clinical pharmacology and drug therapy. Volume 12. 2014. Vol. 3, pp. 13-19;

11. A.T. TePlyakov, S.N.Shilov, A.A. Popova, E.V. GRACOVA, ET AL: State of the cardiovascular system in patients with anthracycline-induced cardiomyopathy // Bulletin of Siberian medicine. 2017; 16 (3): pp. 127-136;

12. A.V. Novik: Anemia and metabolic disorders in cancer patients // Practical oncology. 10. №3.2009. pp. 131-140;

13. Dzhugashvili M., Pokrovsky V. S., Snegovoy A. V. Novel approaches for the correction of mi- cronutrient deficiency in patients with malignant tumors. Malignant Tumours. 2016; 2: 55-65. http:// DOI:10.18027/2224-5057-2016-2-55-65

14. Falanga A., Marchetti M. Hemostatic biomarkers in cancer progression. Thrombosis Research, 2018, V. 164 (Suppl. 1): pp. 54-61;

15. Practical recommendations for the prevention and treatment of thromboembolic complications in cancer patients / Practical recommendations of RUSSCO. Version 2013. - pp. 372-376;

16. Kilickap S, Barista I, Akgul E, Aytemir K, Aksoy S, Tekuzman G. Early and late arrhythmogenic effects of doxorubicin. South Med J. 2007; 100: pp. 262-265. http://doi:10.1097/01. smj.0000257382.89910.fe [ PubMed ]

17. D.N. Kushinskiy, I.V. Tereshina, V.G. Degtyar ET AL: Vascular endothelium growth factor and its receptors in ovarian cancer//Molecular medicine. 2013, pp. 3-11;

18. Lezhnev A., Paramonov V, Solontsov O, Davidov D, Novikov D, Davidov A, Bikbaev R. Endotelial disfunction in The Anaesthesiologists practice//Archiv Euromedica. 2018. Vol.8. №1, pp. 93-96;

19. I. B. Podubna, N.F. Orel: Manual for chemotherapy of tumor diseases. Editor: N. I. Translatorova. M. 2011: pp. 435-436;

20. Volkova M, Russell R. Anthracycline Cardiotoxicity: Prevalence, Pathogenesis and Treatment //Curr Cardiol Rev. 2011 Nov; 7(4): pp. 214-220;

21. E.A. Gaisina, O.V. Nekrasova, V.I. Pavlova: Influence of the preparation of rheamberine on the cardiotoxic effect of anthracyclines in the treatment of locally spread breast cancer // Tyumen Medical Journal. № 3-4. 2010, pp. 41-42;

22. Carvedilol Administration Can Prevent DoxorubicinInduced Cardiotoxicity: A Double-Blind Randomized Trial Tashakori Beheshti A. Mostafavi Toroghi H. Hosseini G. Zarifian A. Homaei Shandiz F. Fazlinezhad A. Cardiology. 2016; 134: 47-53. https://doi. org $/ 10.1159 / 000442722$

23. Y.A. VASYUK, E.L. SHKOLNIK, V.V. NeSVETOV ET AL., Anthracycline cardiotoxicity: prospects of using Ivabradine "Consilium Medicum. Cardiosomatics". 2012. V 3. №4, pp. 65-69;

24. Riad A., Bien S., Westermann D. et al. Pretreatment with statin attenuates the cardiotoxicity of doxorubicin in mice // Cancer Res. 2009. Vol. 69 (2), pp. 695-699;

25. Zakikhani M., Dowling R., Fantus I.G. ET AL. Metformin is an AMPkinase-dependent growth inhibitor for breast cancer cells // Cancer Res. 2006. Vol. 66. P. 10269-10273;

26. Hindler K., Cleeland C. S., Rivera E., ColLARDA. The Role of Statins in Cancer Therapy//The Oncologist. 2006. Vol. 11, pp. 306-315;

27. Bonovas S., Filioussi K., Tsavaris N. et al. Statins and cancer risk: a literature-based meta-analysis and meta-regression analysis of 35 randomized controlled trials // J. Clinic. Oncol. 2006. Vol. 21 (30), pp. 4808-4817; 